\title{
Intraoperative Ultrasound-guided Excision of Cervical Lymph Nodes for Recurrent Differentiated Thyroid Cancer
}

\author{
Magdalena Biggar, Clement Wong, Michael Law, lan Craig Bennett
}

\begin{abstract}
As a result of sensitive thyroglobulin assays and widespread use of cervical ultrasound, endocrine surgeons are encountering patients with impalpable suspicious or frankly metastatic cervical lymph nodes in the follow-up phase after treatment for welldifferentiated thyroid cancer. The surgical excision of impalpable disease of recurrent or persistent thyroid cancer can represent a significant challenge which may require some means of intraoperative localization to ensure that affected nodes are removed. Surgeon-performed intraoperative ultrasound (IOUS) can be used for guiding excision of suspicious but impalpable cervical nodes. The IOUS-guided excision technique is described. The successful application of IOUS to localize and guide resection of impalpable nodal recurrences of papillary thyroid carcinoma in two patients is reported. The utilization of IOUS to guide resection of impalpable pathological nodes in the context of thyroid carcinoma is a safe and practical technique which avoids the need for additional localization procedures and unnecessary potential morbidity for the patient.
\end{abstract}

Keywords: Thyroid carcinoma, Lymph node excision, Recurrence, Ultrasound.

How to cite this article: Biggar M, Wong C, Law M, Bennett IC. Intraoperative Ultrasound-guided Excision of Cervical Lymph Nodes for Recurrent Differentiated Thyroid Cancer. World J Endoc Surg 2013;5(2):45-49.

Source of support: Nil

Conflict of interest: None

\section{INTRODUCTION}

Cervical lymph node metastases are the commonest form of presentation of recurrent well-differentiated thyroid cancer, particularly of the papillary type. Indeed, it has been reported that 5 to $40 \%$ of patients may have either persistent disease or develop recurrent disease in the central or lateral neck nodes. ${ }^{1,2}$ As a result of the utilization of sensitive and stimulated serum thyroglobulin assays, together with more sophisticated cervical imaging, including ultrasound and CT scanning, small impalpable cervical lymph node metastases are increasingly being found in patients being monitored after treatment of a previous thyroid malignancy. Although radioiodine therapy is an option in such circumstances, most specialist thyroid cancer centers acknowledge that best results are achieved with a combination of surgery and radioiodine therapy and would advise removal of all surgically resectable disease prior to radioiodine treatment. ${ }^{3}$ Also, not infrequently recurrent disease may be radioiodine insensitive. This scenario can potentially present technical problems for the endocrine surgeon who may be faced with the task of surgically removing these clinically impalpable nodes or groups of nodes. The majority of previous reports have used invasive techniques, such as hookwire placement or radioisotope injections, to mark the position of the lymph nodes preoperatively which can add significantly to patient morbidity. $^{4-7}$

In this report, we describe the technique of surgeonperformed ultrasound-guided excision of impalpable cervical lymph node metastases. The metastatic or suspicious lymph nodes identified during the course of preoperative workup obviously need to be visible sonographically. The surgeon uses intraoperative ultrasound (IOUS) to localize the involved cervical lymph nodes ontable to ensure that impalpable nodes are accurately excised as part of a compartment-orientated nodal resection, diagnostic procedure or during reoperative surgery.

\section{MATERIALS AND METHODS}

This report describes two patients from one surgeon's practice (IB) who had been previously treated for welldifferentiated thyroid cancer, and who subsequently were found to have metastatic cervical lymph node disease detected by a combination of ultrasonography and fine needle aspiration cytology (FNAC). Both these patients had impalpable cervical disease and their cases illustrate the use of surgeon-performed IOUS to localize and guide surgical excision. Author IB has 17 years experience with ultrasound and has an accredited certificate for clinician performed ultrasound (CCPU) with the Australian Society for Ultrasound in Medicine (ASUM). ${ }^{8}$

In the operating theater, under general anesthesia, the patient was positioned on the table with the neck extended and the skin prepared and draped. Following placement of the ultrasound probe in a sterile sheath, the cervical lymph nodes of interest were localized and the skin was marked in two axes intersecting at the involved site (Fig. 1). A skin incision was then made over or near this area. Once subcutaneous tissues were opened; the ultrasound probe was again placed into the wound to confirm the position of the involved nodes and also to examine the operative field for any additional abnormalities. Aqueous iodine was used as the conducting medium between the sheathed probe and the skin/tissues. A straight mosquito forcep was advanced into the tissues under IOUS guidance, in the same manner 
of performing a fine needle aspiration. The tips of the mosquito were then placed over the affected node and the tissue in this area grasped with Moynihan's forceps so as to anchor the mosquito forcep. The tissues in the vicinity of the mosquito Moynihan's forceps were then excised to encompass the nodal tissues localized by the ultrasound. To ensure adequate clearance of the neck compartment containing the node of interest, additional nodal and fatty tissue was also removed in each instance. Following excision of these tissues, a specimen ultrasound was performed to confirm that the targeted nodes had in fact been removed. Ultrasound was also placed into the surgical site to ensure no sonographic abnormality remained.

\section{RESULTS}

\section{Case 1}

A 42-year-old woman underwent total thyroidectomy and ipsilateral central lymph node dissection for a $23 \mathrm{~mm}$ papillary thyroid cancer in the left lobe. Histopathology identified six lymph nodes which were all noted to contain metastatic carcinoma (one with extranodal spread). The patient underwent Iodine-131 ablation (4,000 MBq) which showed no evidence of residual disease in the neck. Posttherapy (unstimulated) thyroglobulin levels were unrecordable and remained so during further short-term follow-up. Six years after her initial treatment, the patient complained of an alteration in her voice which prompted re-evaluation. Thyroglobulin levels continued to be unrecordable $(<0.5 \mu \mathrm{g} / \mathrm{l})$; however, a neck ultrasound demonstrated two $5 \mathrm{~mm}$ abnormal nodules over the anterior aspect of her neck (level 6, pretracheal region) and a $1.2 \mathrm{~cm}$ suspicious lymph node was also noted in the left lateral region neck (level 4). FNAC from both one of the pretracheal nodes and the lateral neck node showed the presence of

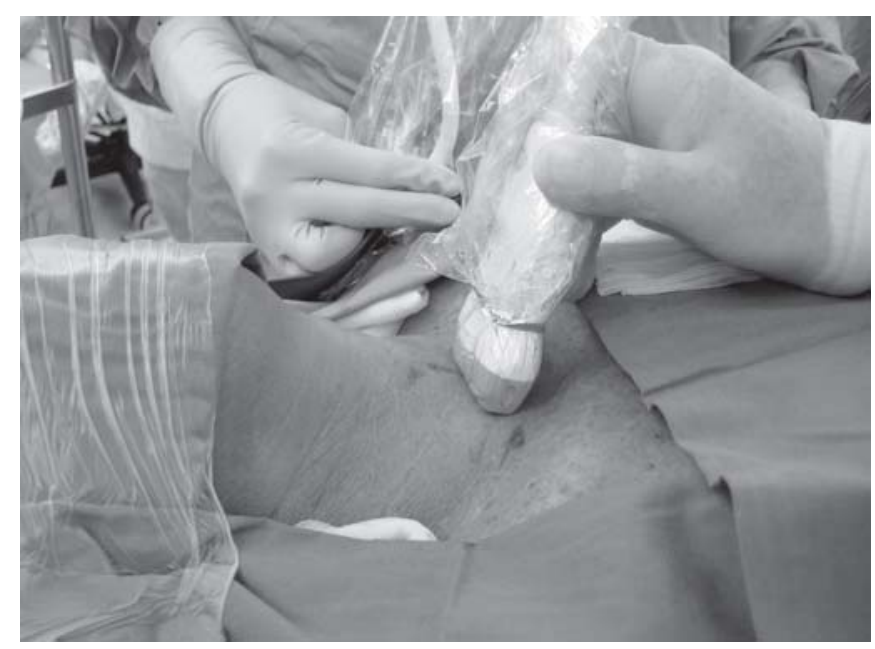

Fig. 1: Performance of on-table IOUS to localize pathological cervical lymph nodes (case 1) metastatic papillary carcinoma. No lymphadenopathy was palpable. A preoperative vocal cord check confirmed normal vocal cord movement and the patient's voice symptom subsequently resolved.

Surgical resection of her neck nodes was performed utilizing intraoperative on-table ultrasound (GE LOGIQ-i portable ultrasound machine) to localize the pathological cervical nodes and to assist in the conduct of a modified neck dissection involving level 6 and 4 compartments. Three of eight lymph nodes contained metastases on final histopathological examination, including two from the pretracheal region. The patient underwent further radioiodine treatment and post-treatment nuclear medicine scans showed no further disease. Post-therapy thyroglobulin levels were unrecordable and the patient has remained free of recurrence after 4 years of subsequent follow-up.

\section{Case 2}

A 38-year-old female underwent a total thyroidectomy and an ipsilateral central neck dissection for a $40 \mathrm{~mm}$ papillary carcinoma in the right lobe of her thyroid gland with two lymph nodes found to contain metastases. The patient was subsequently treated with iodine-131 ablation (4,000 MBq). The post-therapy scan demonstrated residual uptake in her neck suggestive of minor thyroid tissue remnants only with no evidence of residual disease. Although the patient remained asymptomatic, her (unstimulated) serum thyroglobulin estimation was noted to become elevated ( $8.2 \mu \mathrm{g} / \mathrm{l}$; ref $<1.0 \mu \mathrm{g} / \mathrm{l}$ ) approximately 12 months after surgery. While further investigation was recommended, the patient moved interstate and was lost to regular follow-up. The patient was reviewed again 6 years after her original surgery at which time her serum thyroglobulin had risen to $21 \mu \mathrm{g} / \mathrm{l}$. This led to a repeat ultrasound examination of the neck which demonstrated two enlarged lymph nodes over the right side of her neck (Figs 2 and 3). The largest measured $9 \mathrm{~mm}$ and was sited overlying the right carotid sheath (level 3), while the smaller node measured $4 \mathrm{~mm}$ and was noted laterally in level 5. Ultrasound-guided FNAC from the larger precarotid lesion was consistent with metastatic papillary thyroid carcinoma. No cervical lymphadenopathy was palpable.

Through a lateral cervical incision, a modified neck dissection was performed with the aid of intraoperative ultrasound (portable Terason t3000 Ultrasound system) to direct the resection of cervical lymph node compartments. The on-table ultrasound facilitated the identification of the two known abnormal lymph nodes adjacent to and lateral to her carotid sheath, and ensured their removal as part of the resection. These nodes were clearly identified on a postexcision specimen ultrasound scan (Fig. 4). The subsequent histopathology examination identified the 
presence of metastatic papillary carcinoma in the two sonographically identified lymph nodes. No metastatic disease was seen in other lymph nodes examined.

The patient subsequently received further radioiodine ablation therapy (6,000 MBq). No evidence of residual disease was seen on a post-therapy scan and her serum thyroglobulin level subsequently fell to undetectable levels. In both cases, this technique proceeded successfully and uneventfully. Positive identification of the relevant nodes

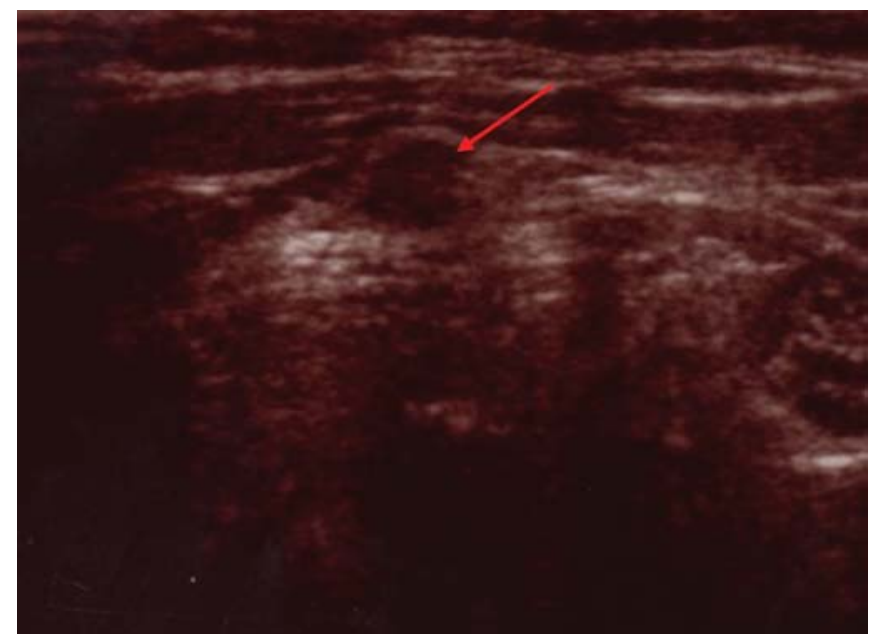

Fig. 2: On-table ultrasound of pathological right lateral cervical lymph node (case 2)

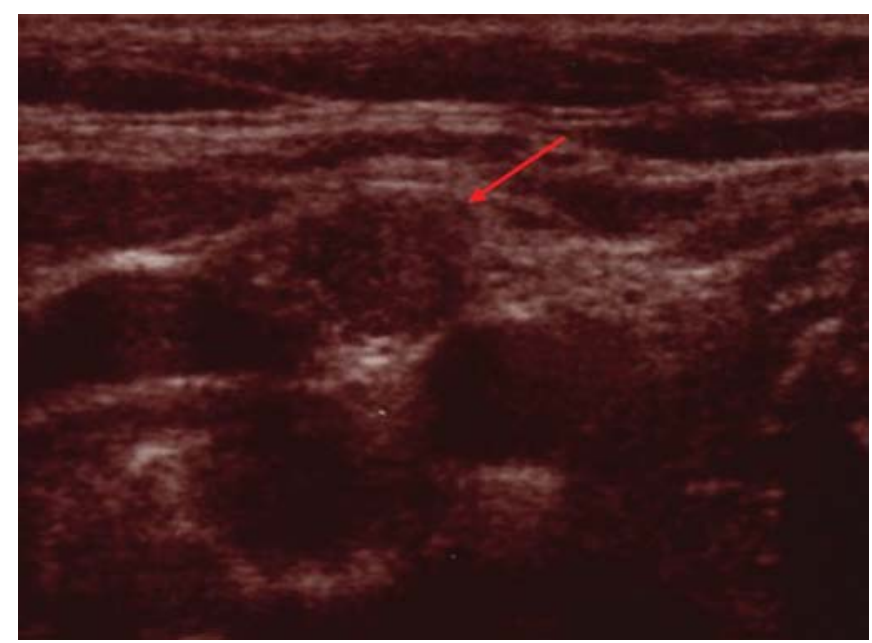

Fig. 3: On-table ultrasound of precarotid pathological lymph node no. 2 (case 2)

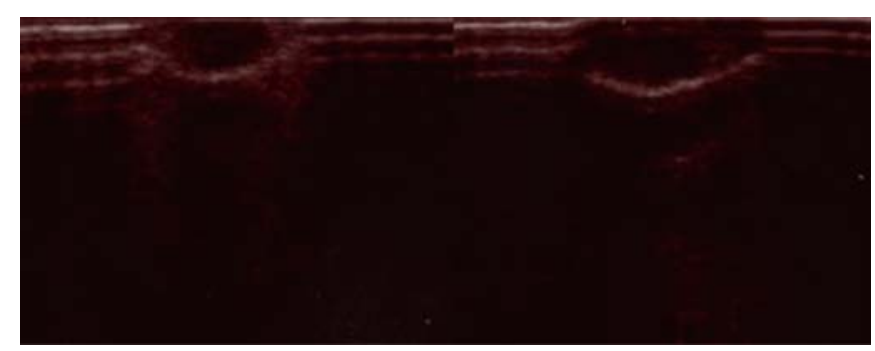

Fig. 4: Specimen ultrasound of $4 \mathrm{~mm}$ lateral cervical lymph node (left) and $9 \mathrm{~mm}$ precarotid lymph node (right) (case 2) was demonstrated in the excised specimens both sonographically and during subsequent histological examination. There was no recurrent laryngeal nerve injury, nor postoperative hypocalcemia in either case.

\section{DISCUSSION}

Endocrine surgeons are being increasingly faced with the scenario of managing patients who have been diagnosed with small impalpable suspicious or frank metastatic lymph node disease in the follow-up phase after treatment for welldifferentiated thyroid cancer. This has arisen as a result of increasingly sensitive thyroglobulin serum assays, a trend to the use of stimulated thyroglobulin, sensitive high frequency ultrasound scanning and FNAC of suspicious sonographic lymph nodes. The sensitivity of FNAC in this setting has also been recently enhanced by the complimentary technique of utilizing a thyroglobulin assay in the washout liquid of the FNA. ${ }^{9}$ The surgical management of these patients can present a technical dilemma for the treating surgeon, as these nodes are often impalpable. Yet it is important that these nodes be accurately surgically targeted as part of any local or compartmental resection which the surgeon feels is appropriate.

There is growing consensus that a formal compartmental neck dissection is indicated for a clinically overt local thyroid cancer recurrence; ${ }^{10-13}$ however, there remains a degree of controversy regarding the management of a subclinical recurrence. Roles for a targeted surgical approach include: ${ }^{14}$ (1) cases when the diagnosis is unable to be definitively proven, such as when a suspicious lymph node lies too close to a major blood vessel to be safe for FNAC ${ }^{15}$ or when FNAC is inconclusive; (2) during a formal selective lateral neck dissection to ensure that the dissection encompasses such small pathological yet impalpable nodes, ${ }^{15}$ (3) in cases of recurrence in a previously operated field, ${ }^{5,15,16}$ (4) in cases of recurrence within a field previously subjected to external beam radiotherapy, ${ }^{17}$ (5) in patients unwilling to 'watch and wait' in the setting of a very small nodal recurrence, and (6) in those wishing to avoid the morbidity of a formal neck dissection. Our two cases illustrated the second and third of these potential scenarios. Previously described operative localizing techniques for targeted lymph node excisions in this setting include: Hookwire-guided excisions, ${ }^{4}$ blue-dye ${ }^{15}$ or charcoal guided localizations ${ }^{16}$ and radio-guided excision. ${ }^{5-7}$ Alternative forms of management include radiofrequency ablation or the use of alcohol. ${ }^{18}$ Advantages and disadvantages of these various methods continue to be debated. ${ }^{16}$ Many of the described surgical techniques have the disadvantage of requiring additional and potentially invasive preoperative interventions, such as hookwire insertions or radioisotope 
injection. Furthermore, the marking method may be inaccurate if the wire dislodges or if dye/isotope is misplaced or permeates through adjacent tissue. Ablative techniques may damage neighboring structures which may lead to serious complications, including vocal cord.

The technique of preoperative hookwire localization is similar to that used for impalpable breast lesions. ${ }^{19}$ The hookwire is usually placed under ultrasound guidance into the neck in the radiology suite and the patient then transferred to the operating room for surgery. However, hookwire-guided placement into the affected nodes is potentially hazardous, should the wire need to be placed adjacent to vital vascular structures and bleeding complications have been reported. ${ }^{20}$ Indeed, proximity to vital structures may preclude this technique from being utilized, as may the presence of multiple or bilateral disease foci. ${ }^{20}$ In addition, the wire may migrate or become dislodged. ${ }^{15}$

The technique of radio-guided surgery is also suboptimal, as the radioisotope injection requires careful timing and, in some techniques, repeated scans up to 12 hours apart..$^{5-7}$ Ironically, the injection itself is usually ultrasound-guided, therefore a seemingly unnecessary additional step is added in the identification process. ${ }^{5}$ In addition, the procedure may be hindered by a variable degree of uptake in the metastatic focus depending on its size and ability to take up radioiosotope, and by the fact that parathyroid glands may also take up the same radioisotope and can therefore be inadvertently removed.

Reports of ultrasound-guided blue dye injection have noted extranodal discoloration in most cases, difficulties when densely calcified nodes are not able to be penetrated by a needle and complications of the injection, such as hematoma and dye allergy. ${ }^{15}$ Charcoal suspension tattoo localization methods have been met with similar problems. ${ }^{16}$

We have described the technique of surgeon-performed IOUS-guided excision of impalpable cervical lymph nodes and its successful application in two cases of recurrent thyroid cancer. Endocrine surgeons are increasingly utilizing and incorporating ultrasound into their day-to-day practice, and many are familiar with office-based ultrasound to review and follow-up thyroid nodules and in the performance of ultrasound-guided FNAC of thyroid lesions or cervical lymph nodes. It is also important that surgeons utilizing ultrasound have appropriate training and credentialing. The Australian Society of Ultrasound in Medicine (ASUM) has now approved a curriculum for endocrine surgeons to receive training and accreditation in the use of ultrasound which includes attending workshops and performing supervised scanning. ${ }^{8}$

The extension of ultrasound usage to the operating theater is therefore not a major step and it is a methodology that most ultrasound-familiar endocrine surgeons could easily adopt. This is a surgical approach which is simple to perform and has been utilized by breast surgeons to remove impalpable breast lesions. ${ }^{21}$ Basic descriptions of the use of ultrasound for the removal of impalpable lymph nodes in recurrent thyroid cancer involved preoperative skin marking only, which had been carried out by radiologists in some reports. ${ }^{22}$ The utilization of ultrasound intraoperatively in the setting of thyroid cancer was described by the Desai and subsequently Karwowski; however, the IOUS examinations were also performed by a radiologist in their series. ${ }^{17,23}$ The technique has gradually appeared elsewhere in the literature, but has neither been described nor discussed in detail. ${ }^{14}$ We feel that the technique deserves further attention, demonstrate that it can be performed by a surgeon and add our refinements to the method of this operative approach.

The major advantage of our technique is the fact that the patient does not require any preparative preoperative intervention nor any invasive procedure. Although at risk structures, such as important nerves in the neck, must be taken into consideration during dissection, regardless of which technique is employed, the advantage of the IOUS technique is that it ensures that abnormal nodes seen on preoperative imaging are indeed excised. Resources required are minimal, given that high frequency portable ultrasound equipment is usually available in the operating room. More than one lesion may be targeted, if required. The use of mosquito and Moynihan forceps as part of the IOUS excision technique acts as an additional guide to the location of the node, yet has the advantage that their placement is under vision within the operative field and avoids preoperative procedures for the patient. A further advantage of the IOUS technique is that immediate confirmation of excision of the relevant nodes can be performed by specimen sonography of the excised tissues. The addition to operative time is minimal and off-set by avoiding preprocedure or preincision interventions required in other techniques. Previous reports have also described that the use of IOUS may identify more disease than what was appreciated during preoperative workup in some cases. ${ }^{17}$

A previous criticism of this method has been that, once subplatysmal flaps are elevated and the dissection begun, the skin marking no longer provides a good reference point to the location of the involved lymph node. ${ }^{15}$ However in our technique, IOUS continues to be used intermittently during the operation, guides the introduction of anchoring forceps and thus continues to guide the dissection. Specific improvements on previous reports which are described in our technique therefore include the surgeon operating the ultrasound, continued intraoperative sonography throughout 
the procedure and the use of forceps to act as an additional ultrasound-guided marker to aid dissection.

\section{CONCLUSION}

The technique of surgeon-performed IOUS-guided excision of impalpable metastatically involved cervical lymph nodes provides the endocrine surgeon with a safe intraoperative tool to accurately target and excise impalpable disease, which minimizes patient discomfort and morbidity. For surgeons already employing office-based ultrasound, the translation of these methods to the operating room can be easily embraced. We believe it is the most appropriate means of surgically managing patients with impalpable lymph node metastases from recurrent thyroid cancer.

\section{REFERENCES}

1. Cady B, Sedgwick CE, Meissner WA, et al. Risk factor analysis in differentiated thyroid cancer. Cancer 1979;42:810-20.

2. Schlumberger MJ. Papillary and follicular thyroid carcinoma. N Engl J Med 1998;338:297-306.

3. Steward DL. Update in utility of secondary node dissection for papillary thyroid cancer. J Clin Endocrinol Metabol 2012;97: 3393-3398.

4. Duprez R, Lebas P, Saint Marc O, et al. Preoperative US-guided hook-needle insertion in recurrent lymph nodes of papillary thyroid cancer: A help for the surgeon. Eur J Radiol 2010;73: 40-42.

5. Erbil Y, Sari S, Agcaoclu O, et al. Radio-guided exicision of metastatic lymph nodes in thyroid carcinoma: A safe technique for previously operated neck compartments. World J Surg 2010; 34:2581-2588.

6. Rubello D, Pelizzo MR, Casara D, et al. Radio-guided surgery for non-131-I-avid thyroid cancer. Thyroid 2006;16:1105-1111.

7. Tükenmez M, Erbil Y, Barbaros U, et al. Radio-guided nonpalpable metastatic lymph node localization in patients with recurrent thyroid cancer. J Surg Oncol 2007;96:534-538.

8. Available from: http://www.asum.com.au/site/index.php. Qualifications; CCPU Certificate in Clinician Performed Ultrasound 2010.

9. Pacini F, Fugazzola L, Lippi F, et al. Detection of thyroglobulin in fine needle aspirates of nonthyroidal neck masses: A clue to the diagnosis of metastatic differentiated thyroid cancer. J Clin Endocrinol Metabol 1992;74:1401-1404.

10. Cooper DS, Doherty GM, Haugen BR, et al. Revised American Thyroid Association management guidelines for patients with thyroid nodules and differentiated thyroid cancer. Thyroid 2009;19:1167-1214.

11. Monchik JM, Delellis RA. Re-operative neck surgery for welldifferentiated thyroid cancer of follicular origin. J Surg Oncol 2006;94:714-718.

12. Sippel RS, Chen H. Controversies in the surgical management of newly diagnosed and recurrent/residual thyroid cancer. Thyroid 2009;19:1373-1380.
13. Solorzano CC, Evans DB. Same-day ultrasound guidance in reoperation for locally recurrent papillary thyroid cancer [comment]. Surgery 2007;142:973-975.

14. Solorzano CC, Carneiro DM, Ramirez M, et al. Surgeonperformed ultrasound in the management of thyroid malignancy. American Surgeon 2004;70:576-582.

15. Sippel RS, Elaraj DM, Poder L, et al. Localization of recurrent thyroid cancer using intraoperative ultrasound-guided dye injection. World J Surg 2009;33:434-439.

16. Hartl DM, Chami L, Al Ghuzlan A, et al. Charcoal suspension tattoo localization for differentiated thyroid cancer recurrence. Ann Surg Oncol 2009;16:2602-2608.

17. Karwowski JK, Jeffrey B, McDougall IR, et al. Intraoperative ultrasonography improves identification of recurrent thyroid cancer. Surgery 2002;132:924-929.

18. Monchik JM, Donatini G, Iannuccilli J, et al. Radiofrequency ablation and percutaneous ethanol injection treatment for recurrent local and distant well differentiated thyroid cancer. Ann Surg 2006;244:296-304.

19. Frank HA, Hall FM, Steer ML. Preoperative localization of nonpalpable breast lesions demonstrated by mammography. N Engl J Med 1976;295:259-260.

20. Triponez F, Poder L, Zarnegar R, et al. Hook needle-guided excision of recurrent differentiated thyroid cancer in previously operated neck compartments: A safe technique for small nonpalpable recurrent disease. J Clin Endocrinol Metab 2006;91: 4943-4947.

21. Bennett IC, Greenslade J, Chiam H. Intraoperative ultrasoundguided excision of nonpalpable breast lesions. World J Surg 2005;29:369-374.

22. McCoy KL, Yim JH, Tublin ME, et al. Same-day ultrasound guidance in reoperation for locally recurrent papillary thyroid cancer. Surgery 2007;142:965-972.

23. Desai D, Jeffrey B, Mcdougall IR, et al. Intraoperative ultrasonography for localization of recurrent thyroid cancer. Surgery 2001;129:498-500.

\section{ABOUT THE AUTHORS}

\section{Magdalena Biggar}

Surgeon, Department of Surgery, Breast and Endocrine Unit Princess Alexandra Hospital, Queensland, Australia

\section{Clement Wong}

Surgeon, Department of Surgery, Breast and Endocrine Unit Princess Alexandra Hospital, Queensland, Australia

\section{Michael Law}

Surgeon, Department of Surgery, Breast and Endocrine Unit Princess Alexandra Hospital, Queensland, Australia

\section{Ian Craig Bennett (Corresponding Author)}

Professor and Surgeon, Department of Surgery, Princess Alexandra Hospital, Queensland, Australia, e-mail: iancben@bigpond.com 